UNIVERSITE DE LAUSANNE - FACULTE DE BIOLOGIE ET DE MEDECINE

Département de radiologie médicale

et

Service de radiodiagnostic et radiologie interventionnelle

\title{
Hepatic Hemangiomas: Factors associated with T2 Shine-Through Effect on Diffusion-Weighted MR Sequences
}

\section{THESE}

préparée sous la direction du Professeur Reto Meuli

et présentée à la Faculté de biologie et de médecine de

l'Université de Lausanne pour l'obtention du grade de

DOCTEUR EN MEDECINE

MI

735

IIUR

\author{
par
Rafael DURAN
BMre 3746 \\ Médecin diplômé de la Confédération Suisse \\ Originaire de Lausanne (Vaud)
}

Lausanne

2014 


\title{
Hepatic hemangiomas: Factors associated with T2 shine-through effect on diffusion-weighted MR sequences
}

\author{
Rafael Duran ${ }^{a, b, *}$, Maxime Ronot ${ }^{b, c}$, Anne Kerbaol ${ }^{b}$, \\ Bernard Van Beers ${ }^{b}$, Valérie Vilgrain ${ }^{\mathrm{b}, \mathrm{c}}$ \\ 'Centre Hospitalier Universitaire Vaudois and University of Lausanne, Department of Radiology, Lausanne, Switzerland \\ b Assistance-Publique Hopitaux de Paris, APHP, Hôpital Bequjon, Department of Radiology, Clichy, France \\ ' University Paris Diderot, Sorbonne Paris Cité, INSERM U773, centre de recherche biomédicale Bichat-Beaujon, CRB3, Paris, France
}

\section{A R T I C L E I N F O}

\section{Article history:}

Received 6 August 2013

Accepted 27 November 2013

\section{Keywords:}

Hemangioma

Benign liver lesion

Diffusion magnetic resonance imaging

MRI

Imaging

Diagnosis

\begin{abstract}
A B S T R A C T
Purpose: To determine the frequency and factors associated with the presence of T2 shine-through effect in hepatic hemangiomas on diffusion-weighted (DW) magnetic resonance (MR) sequences.

Materials and methods: This retrospective study was approved by institutional review board with waiver of informed consent. One hundred forty-nine consecutive patients with 388 hepatic hemangiomas who underwent a liver MR between January 2010 and November 2011 were included. MR analysis evaluated the lesion characteristics (signal intensities and enhancement patterns (classical, rapidly filling, delayed filling)), the presence of T2 shine-through effect on DW sequences ( $b$ values of 0,150 , and $600 \mathrm{~s} / \mathrm{mm}^{2}$ ), and apparent diffusion coefficient $(A D C$ ) values. Multivarjate analysis was performed to study the factors associated with the T2 shine-through effect.

Results: T2 shine-through effect was observed in 204/388 (52.6\%) of hepatic hemangiomas and in 100 (67.1\%) patients. Mean ADC value of hemangiomas with T2 shine-through effect was significantly lower than hemangiomas without $(2.0 \pm 0.48$ vs $2.38 \pm 0.45, P<.0001)$. On multivariate analysis, high signal intensity on fat-suppressed $\mathrm{T} 2$-weighted fast spin-echo images, hemangiomas with classical or delayed enhancement, and the ADC of the liver were the only significant factors associated with $\mathrm{T} 2$ shine-through effect.

Conclusion: T2 shine-through effect is commonly observed in hepatic hemangiomas and is related to hemangiomas characteristics. Radiologists should be aware of this phenomenon which could lead to misdiagnosis. Its presence should not question the diagnosis of hemangiomas when typical MR findings are found.
\end{abstract}

(C) 2013 Elsevier Ireland Ltd, All rights reserved.

\section{Introduction}

Hepatic hemangiomas are the most common benign hepatic tumors and their prevalence ranges from 0.4 to $20 \%$ [1,2]. Hepatic hemangiomas are commonly found incidentally at routine radiologic examinations such as sonography and computed tomography (CT) [2,3]. MR imaging is indicated for lesion characterization when sonography or CT are inconclusive or when liver lesions are seen in patients with chronic liver disease or in oncologic patients. MR imaging has been shown as the best imaging modality for diagnos-

Abbreviations: DW, diffusion-weighted; $M R$, magnetic resonance; $A D C$, apparent diffusion coefficient; CT, computed tomography; ROI, region of interest.

* Corresponding author at: University Hospital of Lausanne (CHUV), Department of Radiology, Rue du Bugnon 46, 1011 Lausanne, Switzerland. Tel.: +14436823781. E-mail addresses: Rafaelduran@chuvch, rduran4iijhmi.edu (R. Duran), Maxine,ronotobjn.zphp.fr(M. Ronot), Annekerbalobjn.aphph (A. Kerbaol),

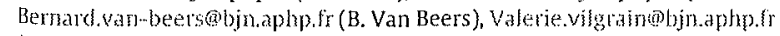
(V. Vilgrain). ing hepatic hemangiomas with high sensitivity and specificity ( $84 \%$ and $100 \%$, respectively) [4]. Typical hemangiomas are hypointense on T1-weighted MR images and markedly hyperintense on T2weighted images. On contrast-enhanced images, they demonstrate early peripheral nodular enhancement with progressive centripetal enhancement on subsequent images [2,5]. MR imaging is also helpful for diagnosing atypical hemangiomas [6-8].

Diffusion-weighted (DW) MR images are useful for characterizing focal hepatic lesions and can help to distinguish between benign and malignant lesions $[9,10]$. Then a lesion can be considered benign (mostly cyst and hemangioma) if the lesion is hyperintense on T2-weighted images and on DW images at $b=0 \mathrm{~s} / \mathrm{mm}^{2}$, with a strong signal intensity decrease at high $b$ values and an $A D C$ that is subjectively higher than that of the liver [9]. Using these criteria, Parikh et al. showed that the overall accuracy of DW images was slightly better than that of T2-weighted images [9].

It is commonly considered that while most hepatic hemangiomas typically show suppression of high signal intensity at high $b$ values (i.e. $500-750 \mathrm{~s} / \mathrm{mm}^{2}$ ), some may demonstrate residual high

0720-048X/\$ - see front matter $\odot 2013$ Elsevier Ireland Ltd. All rights reserved.

htip://dx.doi.org/10.1016/j, ejlad.2013.11.023 


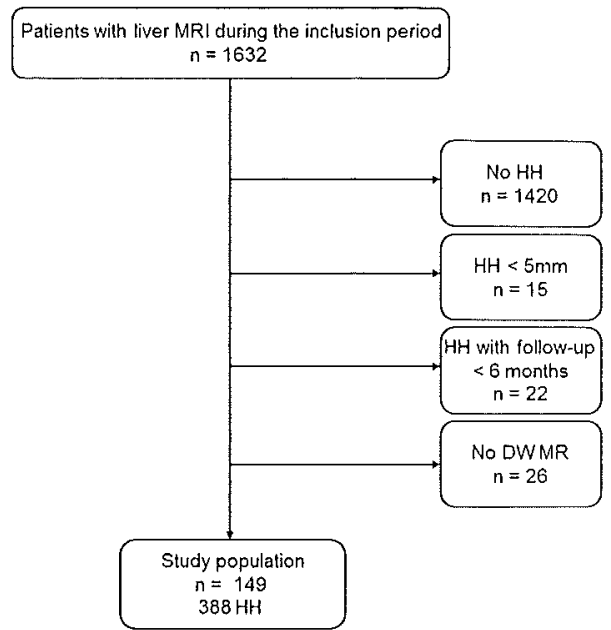

Fig. 1. Flowchart illustrates patient selection. $M R I=M R$ imaging, $H H=$ hepatic hemangioma.

signal intensity on high $b$ value images and are therefore difficult to characterize with visual assessment of the DW MR images $[10,11]$. This phenomenon is called T2 shine-through effect and was first observed in brain diffusion imaging, more particularly in the evaluation of acute cerebral ischemia [12]. It is attributed to the contribution of $\mathrm{T} 2$ hyperintensity on the overall signal intensity of DW MR images. Indeed, signal intensity of DW MR images is composed of mixed contributions from the intrinsic $T 2$ properties of the tissue being examined, the $A D C$, and to a lesser degree, of the spin density [12]. T2 shine-through effect may occasionally be encountered in benign liver tumors, but little is known about this effect in hepatic hemangiomas [10].

Thus, the purpose of this study was to determine the frequency and associated factors of $T 2$ shine-through effect in a large series of patients with hepatic hemangiomas.

\section{Materials and methods}

This retrospective single-center study was conducted in a tertiary hospital for abdominal diseases and was approved with a waiver of informed consent by the institutional review board of the same hospital.

\subsection{Study design}

Patients identified in the database of the tertiary hospital mentioned above for MR imaging reports who underwent MR imaging of the liver with a DW sequence between January 2010 and November 2011 and who had at least one hepatic hemangioma measuring at least $5 \mathrm{~mm}$ in minimum diameter were included. DW sequences were performed as part of our routine liver imaging protocol.

A total of 1632 patients were identified. One thousand four hundred eighty-three patients were excluded for the following reasons: no lesion suggesting hepatic hemangioma $(n=1420)$, hepatic hemangioma measuring less than $5 \mathrm{~mm}$ in diameter $(n=15)$, hepatic hemangioma with follow-up imaging of less than 6 months $(n=22)$, no DW sequences on the picture archiving and communication system $(n=26)$ (Fig. 1$)$. On the basis of these criteria, the final study population included 149 patients (mean age, 52.5 years; range, 22-84 years) with 388 hemangiomas and of these, 74 (49.7\%) were women (mean age, 50.6 yêrs; range, 24-84 years) and 75 $(50.3 \%)$ were men (mean age, 54.3 years; range, $22-78$ years).
Hepatic hemangiomas were solitary in 67 patients (45\%) and multiple in 82 (55\%); there were 2 lesions in 30 patients, 3 lesions in 21 patients, 4 lesions in 10 patients, 5 lesions in 7 patients and 6 or more lesions in 14 patients.

\subsection{Reference standard}

Diagnosis of hemangiomas was established based on a combination of typical imaging features and follow-up imaging showing at least 6 months of stability or on histological analysis. MR imaging features were considered for the diagnosis of hemangioma $[5,6,13]$ : well demarcated and notably hyperintense on heavily T2-weighted images, and (a) peripheral globular discontinuous enhancement over time on contrast-enhanced dynamic images with progressive and centripetal enhancement; or (b) immediate homogeneous enhancement and iso or hyperintensity compared with surrounding liver parenchyma at the equilibrium phase.

Three hundred seventy-seven hemangiomas met these criteria (97.2\%). All hepatic hemangiomas remained stable over time. Because some of our patients had previous MR examinations, the median follow up period was 18.6 months (range 6-81 months).

The remaining hepatic hemangiomas $(11 / 388,2.8 \%)$ did not meet the imaging criteria and the diagnosis required histologic confirmation which was obtained by means of biopsy. Eight hemangiomas were isointense on T2-weighted images and three hemangiomas did not enhance on contrast-enhanced sequences.

\subsection{MR imaging}

Patients were examined with a 1.5-T superconducting MR system using a phased-array surface coil for signal reception. The protocol included a T2-weighted fast spin-echo sequence with spectral fat saturation and a T2-weighted single-shot sequence, a T1-weighted dual fast gradient-recalled echo sequence, and a transverse breath-hold three-dimensional T1-weighted fatsuppressed spoiled gradient-recalled echo sequence before and after dynamic injection of $0.1 \mathrm{mmol}$ per kilogram of body weight of non-specific gadolinium chelates, followed by a $20-\mathrm{mL}$ saline solution flush at a rate of $2 \mathrm{~mL} / \mathrm{s}$ administered with a power injector. Hepatic arterial-dominant, portal venous, and equilibrium phase sequences were performed 20-25s,60-70s, and 180-200s after intravenous contrast material injection, respectively. Delayed phase sequence (obtained 6-10 min after the administration of contrast material) was not part of our systematic protocol and was performed in 28 patients with 67 hemangiomas. The other parameters are summarized in Table 1 .

\subsection{DW MR protocol}

A free-breathing fat-suppressed single-shot echoplanar DW MR sequence was performed before contrast material injection in the transverse plane with tridirectional diffusion gradients (being collinear with the Cartesian axes of the scanner coordinate system) to obtain images sensitive to diffusion. Gradient strengths corresponding to $b$ values of 0,150 , and $600 \mathrm{~s} / \mathrm{mm}^{2}$ were used with trace (average) image assessment. Fat suppression was implemented by the spectral presaturation with inversion recovery technique. Cardiac gating was not used. No antispasmodic drugs were administered. An ADC map was generated between T2 echoplanar images and the trace image by using the three-point method. The other parameters are summarized in Table 1.

\subsection{Image analysis}

The study coordinator (a radiologist with 7 years of experience) reviewed all MR examinations of the study period and collected 
rable 1

MR sequence parameters.

\begin{tabular}{|c|c|c|c|c|c|}
\hline \multirow{2}{*}{$\begin{array}{l}\text { Parameter } \\
\text { Sequence }\end{array}$} & \multicolumn{2}{|c|}{ T2-weighted imaging } & \multirow{2}{*}{$\begin{array}{l}\text { T1-weighted imaging } \\
\text { Dual fast GRE }\end{array}$} & \multirow{2}{*}{$\begin{array}{l}\text { DW MR imaging } \\
\text { Echoplanar }\end{array}$} & \multirow{2}{*}{$\begin{array}{l}\text { Dynamic CE imaging } \\
\text { 3D spoiled GRE }\end{array}$} \\
\hline & Fast spin echo & Single-shot & & & \\
\hline Respiratory triggered & Yes & No & No & No & No \\
\hline Fat suppression & Yes (SPIR) & No & No $(I P / O P)$ & Yes (SPIR) & Yes (water selection) \\
\hline Field of view $(\mathrm{cm})$ & $30-40 \times 30-40$ & $30-40 \times 30-40$ & $30-40 \times 30-40$ & $30-40 \times 30-40$ & $30-40 \times 30-40$ \\
\hline Matrix (phase $\times$ frequency encoding) & $192 \times 256$ & $192 \times 256$ & $168 \times 256$ & $120 \times 256$ & $192 \times 256$ \\
\hline Repetition time (ms) & $1600-8000$ & $11,351 / 19,173$ & 235 & 3448 & $3.3-4.7$ \\
\hline Echo time (ms) & 90 & 85 & $2.3 / 4.6$ & 63 & $1.4-2.3$ \\
\hline Flip angle (") & 90 & 90 & 80 & 90 & 10 \\
\hline Echo planar imaging factor & - & - & - & $45-51$ & - \\
\hline Parallel imaging acceleration factor & 2 & 2 & 2 & 2 & 2 \\
\hline Number of signals acquired & 1 & 1 & 1 & 8 & 1 \\
\hline Section thickness (mm) & $4-5$ & 5 & 5 & 6 & 4 \\
\hline Intersection gap ( $\mathrm{mm}$ ) & 1 & $1-1.5$ & 1 & 0.6 & -2 \\
\hline Number of sections & $32-35$ & 32 & 32 & 32 & 32 \\
\hline$b$ values $\left(\mathrm{s} / \mathrm{mm}^{2}\right)$ & - & - & - & $0,150,600$ & - \\
\hline Acquisition time & $2-3 \mathrm{~min}$ & $19-23 \mathrm{~s}$ & $16-20 s$ & $4-5 \min$ & $20-25 \mathrm{~s}$ \\
\hline
\end{tabular}

Acquisition time

Note: $\mathrm{SPIR}=$ spectral presaturation with inversion

CE $\mathrm{C}=$ contrast-enhanced, $3 \mathrm{D}=$ three-dimensional.

imaging findings of patients who fulfilled inclusion criteria. The images were evaluated by two radiologists (R.D. and M. R., with 7 and 10 years of experience). Readers were blinded to clinical information including pathological conditions and previous imaging. All sequences including DW MR images were read in the same session. The MR characteristics of hemangiomas were determined in consensus. Lesions which met the imaging criteria of a cyst were not evaluated.

\subsection{Analysis of conventional MR images}

The size and location determined according to the Couinaud classification were recorded for each patient. The signal intensity of all the hemangiomas was graded on T2-weighted and T1-weighted images as isointense, hypointense, or hyperintense in relation to normal liver tissue. High signal intensity lesions on T2-weighted images were also compared to the spleen and low signal intensity lesions on T1-weighted images were compared to the aorta.

On dynamic contrast-enhanced MR sequences, hemangiomas were classified as follows: (i) classical if hemangioma shows early, peripheral, globular, and discontinuous enhancement with progressive centripetal and prolonged enhancement, (ii) rapidly filling if hemangioma shows immediate homogeneous enhancement at the arterial phase and iso or hyperintensity compared with surrounding liver parenchyma at the equilibrium phase [7,8], (iii) delayed filling if hemangioma shows a lack of enhancement in the arterial phase with bright-dot enhancement or minimal peripheral enhancement in the portal or the equilibrium phase [14].

Liver steatosis was searched and defined as a decrease in signal intensity in the opposed-phase gradient-recalled echo T1-weighted MR sequence.

\subsection{Analysis of DW MR images}

Image quality of DW MR images was assessed on a three-point scale (good, satisfactory, unsatisfactory). Lesions were analyzed on images with a $b$ value of 0,150 , and $600 \mathrm{~s} / \mathrm{mm}^{2}$ for signal intensity relative to the liver and presence of $\mathrm{T} 2$ shine-through effect. The SI of the lesions was graded as isointense, hypointense, or hyperintense in relation to liver parenchyma. To reflect clinical practice, the presence of $\mathrm{T} 2$ shine-through effect was assessed qualitatively and $\mathrm{T} 2$ shine-through effect was considered present when the lesion-to-liver contrast was similar or increased with a $b$ value of $600 \mathrm{~s} / \mathrm{mm}^{2}$ compared with a $b$ value of $0 \mathrm{~s} / \mathrm{mm}^{2}$, and absent if the lesion-to-liver contrast was decreased with a $b$ value of $600 \mathrm{~s} / \mathrm{mm}^{2}$ compared with a $b$ value of $0 \mathrm{~s} / \mathrm{mm}^{2}$. In heterogeneous hepatic hemangiomas on DW MR images, a T2 shine-through effect was considered present when the lesion-to-liver contrast was similar or increased anywhere in the lesion with a $b$ value of $600 \mathrm{~s} / \mathrm{mm}^{2}$.

ADC values of lesions were obtained from pixel-based ADC maps. The software provided by the superconducting MR system was used for calculating ADC maps. The largest possible region of interest (ROI) was drawn for each tumor in order to encompass as much of the lesion as possible (mean surface, $2.4 \mathrm{~cm}^{2}$; range, $0.16-135.4 \mathrm{~cm}^{2}$ ). The ROIs were placed at a level of section on which the lesion had its largest diameter. The ROIs created on the trace images were superimposed on the ADC map. The location of lesions that were not visualized on DW images was determined by using T2-weighted and/or contrast-enhanced T1-weighted images. Three ROIs of at least $1 \mathrm{~cm}^{2}$ (mean, $1.9 \mathrm{~cm}^{2}$; range, $1-2.8 \mathrm{~cm}^{2}$ ) were placed in the adjacent normal appearing liver parenchyma in the same lobe as the lesion and ADC values were then averaged. ROI placement was carefully performed to avoid any adjacent main branch blood vessels, the gallbladder, liver periphery, and motion artifacts.

\subsection{Statistical analysis}

Hemangioma characteristics (size, location, signal intensities on T2- and T1-, enhancement patterns, and ADC values) as well as liver steatosis were compared between the group of hemangiomas with $\mathrm{T} 2$ shine-through effect and the group without. Continuous variables were tested by using the Mann-Whitney-Wilcoxon test. Fisher's exact test was used in the analysis of contingency tables. Multivariate analysis was performed by using JMP, Version 9.0, SAS Institute Inc., Cary, NC. A difference with a $P$ value of less than .05 was considered statistically significant. In patients with multiple lesions, the analysis was not performed by using cluster mode because it has been previously shown that hemangioma characteristics may differ in the same patient [15].

\section{Results}

\subsection{Qualitative analysis}

The mean size of hemangiomas was $18.8 \pm 19.2 \mathrm{~mm}$ ranging from 5 to $150 \mathrm{~mm}$. Sixty-two hemangiomas were located in the left liver lobe (i.e. segment 2 and 3), none in the caudate lobe, 38 in the segment 4, 107 and 181 in the right anterior (i.e. segment 5 and 8 ) and posterior (i.e. segment 6 and 7 ) segments, respectively. 


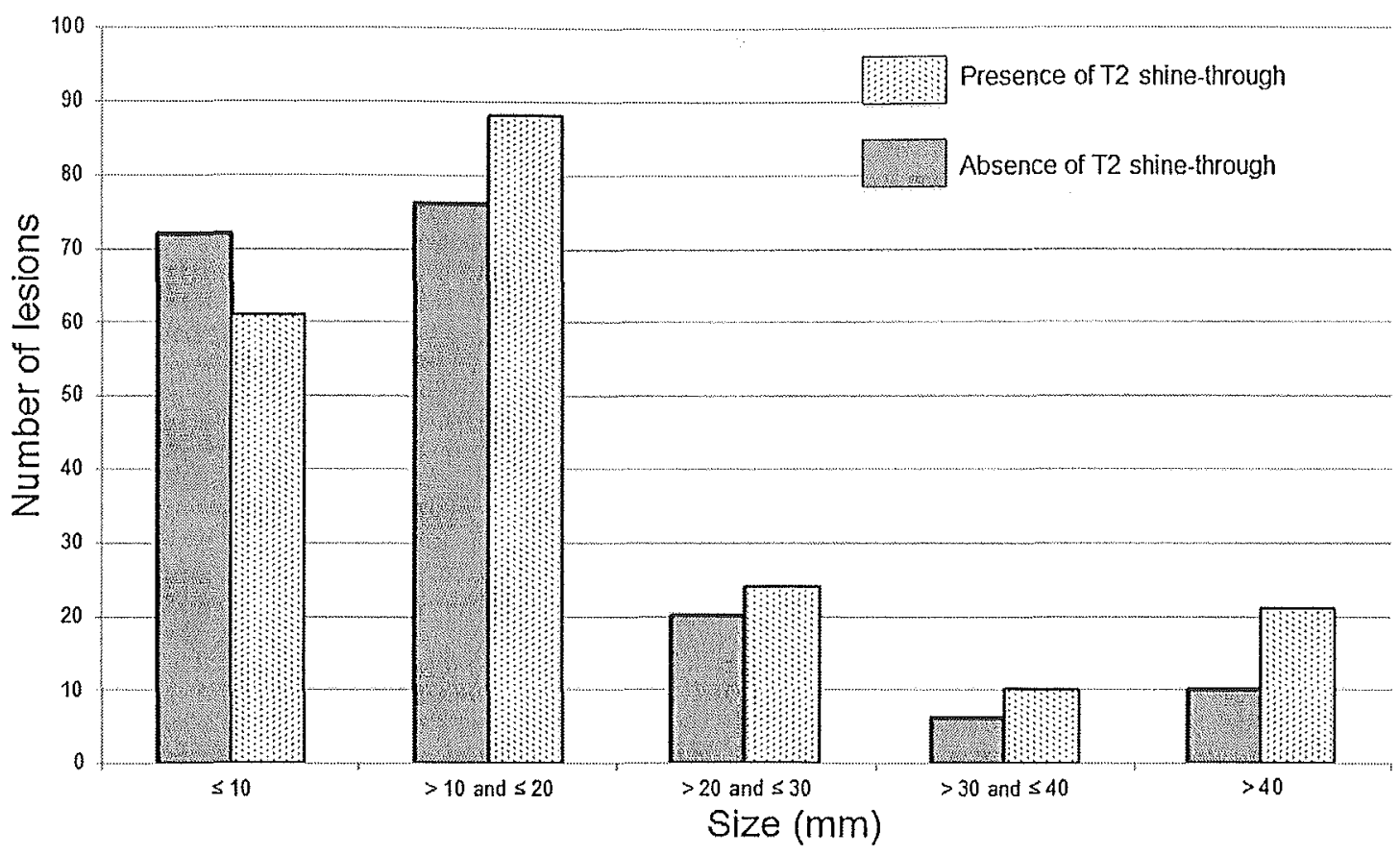

Fig. 2. Presence or absence of $\mathrm{T} 2$ shine-through effect according to the size of the hepatic hemangiomas.

On fat-suppressed $\mathrm{T} 2$-weighted fast spin-echo sequences, nearly all hemangiomas were hyperintense relative to liver and to spleen $(n=360(92.8 \%))$ or to liver alone $(n=26(6.7 \%))$. On single-shot 'T2-weighted sequences, nearly all hemangiomas were hyperintense relative to liver and to spleen $(n=355(91.5 \%)$ ) or to liver alone $(n=25(6.4 \%))$. Only two $(0.5 \%)$ and eight (2.1\%) hemangiomas were isointense relative to liver on fat-suppressed T2-weighted fast spin-echo and on single-shot T2-weighted sequences, respectively.

On in-phase gradient-recalled echo T1-weighted images, nearly all hemangiomas were hypointense relative to liver and to aorta (similar or lower than aorta) $(n=181(46.6 \%))$ and relative to liver alone $(n=200(51.6 \%))$. Only seven ( $1.8 \%)$ hemangiomas were isointense relative to liver. On opposed-phase gradient-recalled echo T1-weighted images, liver steatosis was present in 29/149 patients (19.5\%).

On three-dimensional $\mathrm{T} 1$-weighted fat-suppressed spoiled gradient-recalled echo contrast-enhanced sequences, hepatic hemangiomas had classical enhancement pattern in 266/388 patients $(68.5 \%)$, whereas rapidly filling and delayed filling enhancement patterns were present in $62 / 388$ patients (16\%) and $60 / 388$ patients (15.5\%), respectively.

All DW MR images were of diagnostic quality. T2 shine-through effect was observed in 204/388 (52.6\%) of hepatic hemangiomas and in $100(67.1 \%)$ patients. The mean size of hepatic hemangiomas showing T2 shine-through effect was $22 \mathrm{~mm}$ whereas it was of $16 \mathrm{~mm}$ in lesions that did not exhibit $\mathrm{T} 2$ shine-through effect $(P=.0455)$. Fig. 2 summarizes the presence or absence of $\mathrm{T} 2$ shinethrough effect according to the size of the hepatic hemangiomas.

$\mathrm{T} 2$ shine-through effect was more frequent in hemangiomas in the right liver lobe than in hemangiomas in the left liver lobe ( $n=179 / 326(54.9 \%)$ vs $n=25 / 62(40.3 \%), P=.0381$, respectively). No statistical difference was found when comparing hemangiomas in the left vs right livers.

T2 shine-through effect was observed more frequently in patients with hemangiomas having hyperintensity similar or superior to the spleen on fat-suppressed T2-weighted fast spin-echo sequences, than in the other patients $(197 / 360(54.7 \%)$ vs $7 / 28$
(25\%), $P=.0028$, respectively). This was not significant with singleshot T2-weighted sequences. T2 shine-through effect was more frequently observed in patients with hemangiomas having very low lesion intensity (similar or lower than aorta) on in-phase gradientrecalled echo $\mathrm{T} 1$-weighted images than in the other patients $(n=106 / 181$ (58.6\%) vs $n=98 / 207$ (47.3\%), $P=.0249$, respectively). The presence of the $T 2$ shine-through effect was not associated with liver steatosis.

T2 shine-through effect was observed in classical, rapidly filling, and delayed filling hemangiomas in 151/266 (56.8\%) (Figs. 3 and 4 ), $15 / 62$ (24.2\%), and $36 / 60(60 \%)$ (Fig. 5), respectively $(P<.0001$ all together, with significant differences between classical and rapidly filling hemangiomas $(P<.0001)$ and between delayed filling and rapidly filling hemangiomas $(P<.0001)$ ) (Fig. 6 ).

T2 shine-through effect was more frequent in typical hemangiomas (defined as lesion signal intensity similar or stronger than spleen on both fat-suppressed T2-weighted fast spin-echo and single-shot T2-weighted sequences, and classical contrast enhancement) than in the others $(144 / 248(58.1 \%)$ vs $60 / 140$ (42.9\%), $P=.0043$ ) (Fig. 7).

\subsection{Quantitative analysis}

The mean ADC value of all hemangiomas was significantly higher than that of the liver $\left(2.17 \pm 0.36 \times 10^{-3} \mathrm{~mm}^{2} / \mathrm{s}\right.$ vs $1.48 \pm 0.21 \times 10^{-3} \mathrm{~mm}^{2} / \mathrm{s}$, respectively, $\left.P<.0001\right)$. The mean $A D C$ value of hemangiomas in the right liver was significantly lower than that in the left liver $\left(2.12 \pm 0.32 \times 10^{-3} \mathrm{~mm}^{2} / \mathrm{s}\right.$ vs $2.32 \pm 0.42 \times 10^{-3} \mathrm{~mm}^{2} / \mathrm{s}$, réspectively, $P=.0021$ ). Mean $\mathrm{ADC}$ value of hemangiomas showing $\mathrm{T} 2$ shine-through effect was significantly lower than hemangiomas without $\mathrm{T} 2$ shine-through effect $\left(2.00 \pm 0.48 \times 10^{-3} \mathrm{~mm}^{2} / \mathrm{s}\right.$ vs $\left.2.38 \pm 0.45 \times 10^{-3} \mathrm{~mm}^{2} / \mathrm{s}, P<.0001\right)$. Mean ADC value of adjacent liver was significantly lower in patients with hemangiomas showing T2 shine-through effect than in others $\left(1.44 \pm 0.23 \times 10^{-3} \mathrm{~mm}^{2} / \mathrm{s}\right.$ vs $1.53 \pm 0.32 \times 10^{-3} \mathrm{~mm}^{2} / \mathrm{s}$, $P=.0214)$. Mean ADC value of the hemangiomas according to the classical, rapidly filling and delayed filling enhancement pattern was significantly different $\left(2.17 \pm 0.48 \times 10^{-3} \mathrm{~mm}^{2} / \mathrm{s}\right.$, 

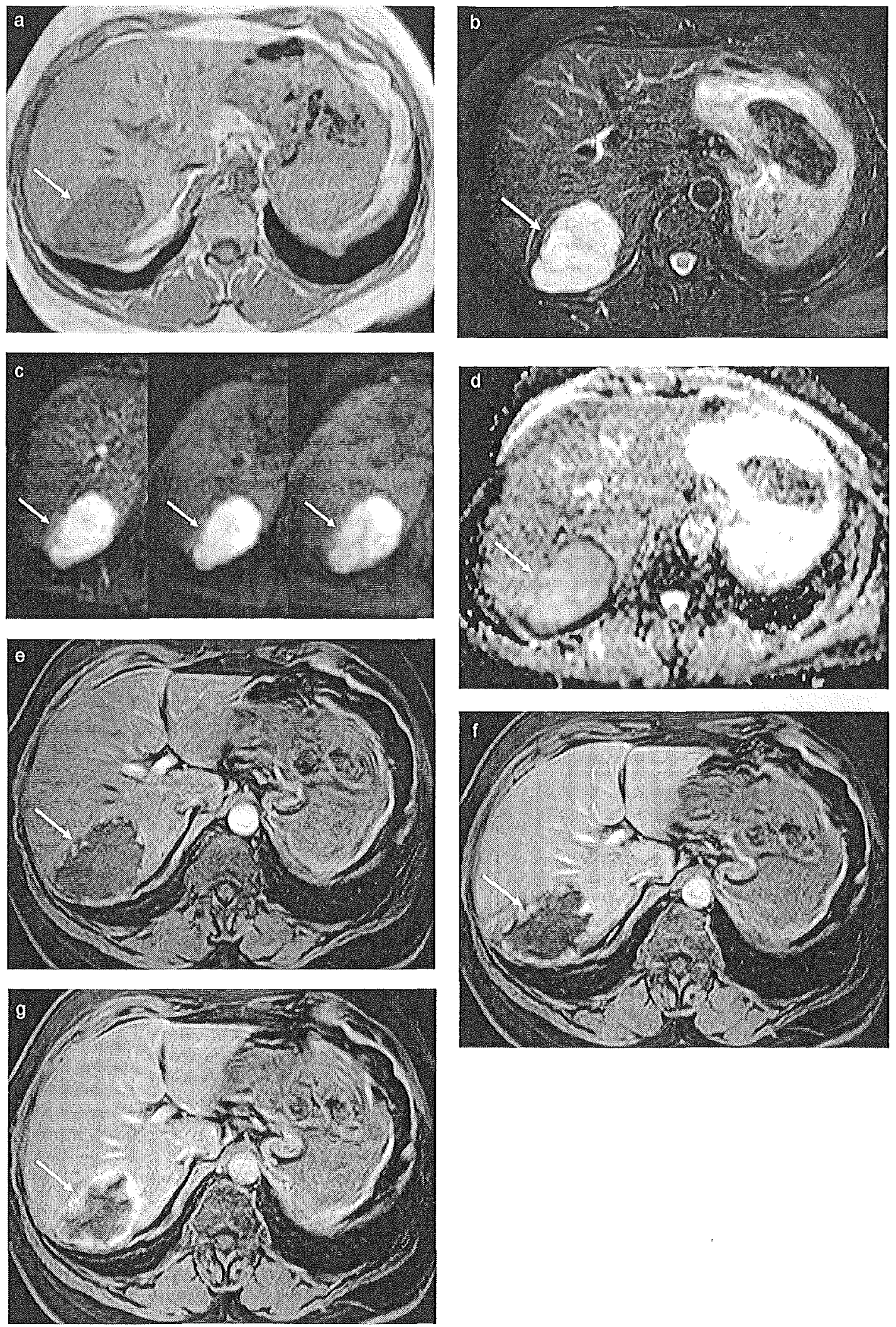

Fig. 3. Hepatic hemangioma with classical enhancement pattern in a 55-year-old woman with unknown hepatic lesion on ultrasound. (a) On in-phase gradient-recalled echo T1-weighted MR sequence, the hepatic hemangioma appears hypointense (arrow) and (b) on fat-suppressed T2-weighted fast spin-echo MR sequence, the lesion is hyperintense relative to surrounding liver and spleen (spleen not shown) (arrow). (c) On DW MR sequence, the lesion-to-liver contrast is similar with a $b$ value of $600 \mathrm{~s} / \mathrm{mm}^{2}$ compared with a $b$ value of $0 \mathrm{~s} / \mathrm{mm}^{2}$ meaning $\mathrm{T} 2$ shine-through effect (arrows). (d) ADC value of the lesion is $1.69 \pm 0.34 \times 10^{-3} \mathrm{~mm} / 2 / \mathrm{s}$ (arrow). (e-g) On dynamic contrast-enhanced MR sequences, the lesionshows early, peripheral, globular, and discontinuous enhancement with progressive centripetal enhancement (arrows). 

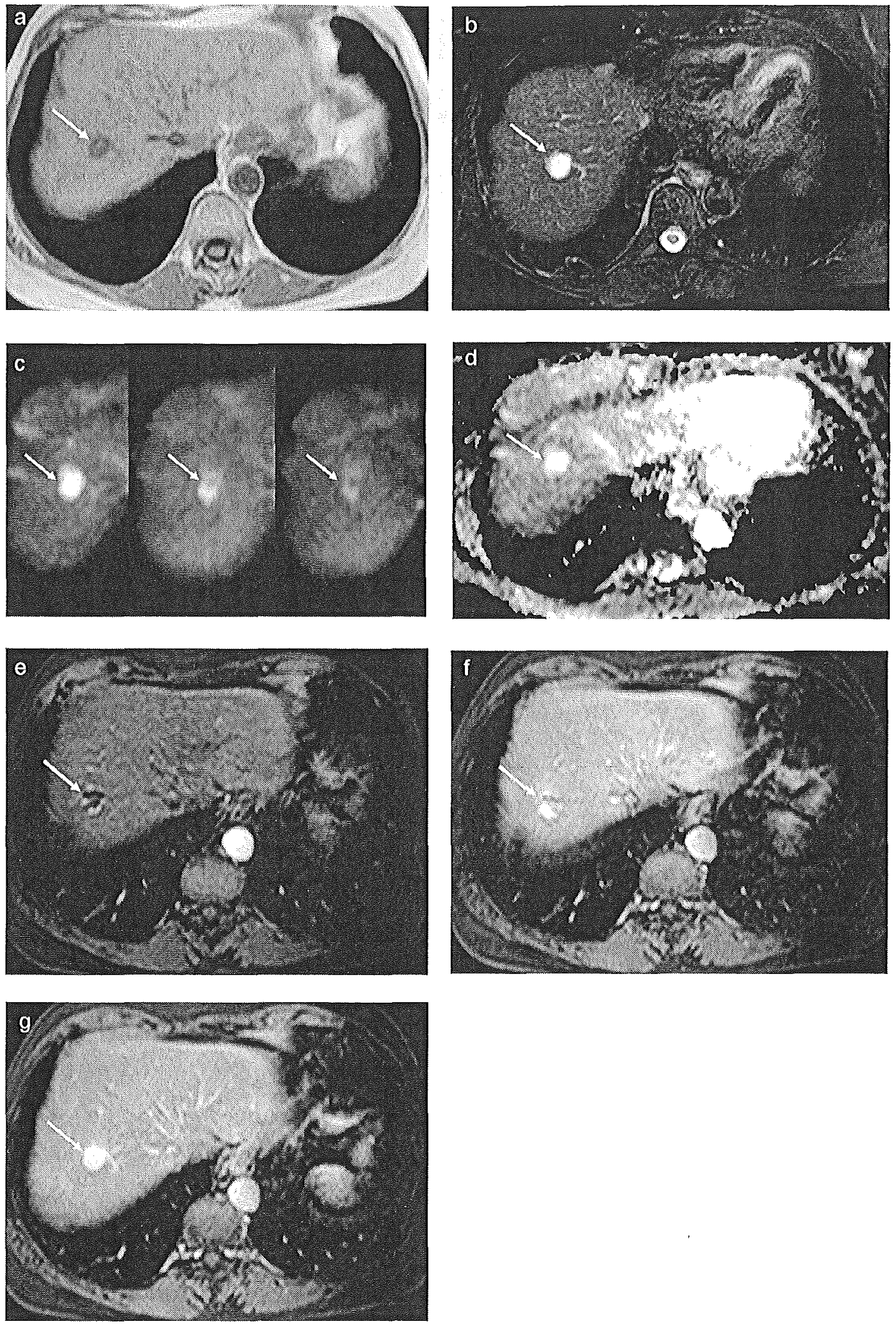

Fig. 4. Hepatic hemangioma with classical enhancement pattern in a 67-year-old woman with breast cancer. (a) On in-phase gradient-recalled echo T1-weighted MR sequence, the hepatic hemangioma appears hypointense (arrow) and (b) on fat-suppressed T2-weighted fast spin-echo MR sequence, the lesion is hyperintense relative to surrounding liver and spleen (spleen not shown) (arrow). (c) On DW MR sequence, the lesion-to-liver contrast is decreased with a $b$ value of $600 \mathrm{~s} / \mathrm{mm}^{2} \mathrm{compared}$ with a $b$ value of $0 \mathrm{~s} / \mathrm{mm}^{2}$ (absence of T2 shine-through effect) (arrows). (d) ADC value of the lesion is $2.89 \pm 0.42 \times 10^{-3} \mathrm{~mm}^{2} / \mathrm{s}$ (arrow). (e-g) On dynamic contrast-enhanced $\mathrm{MR}$ sequences, the lesion shows early, peripheral, globular, and discontinuous enhancement with progressive centripetal enhancement (arrows). 

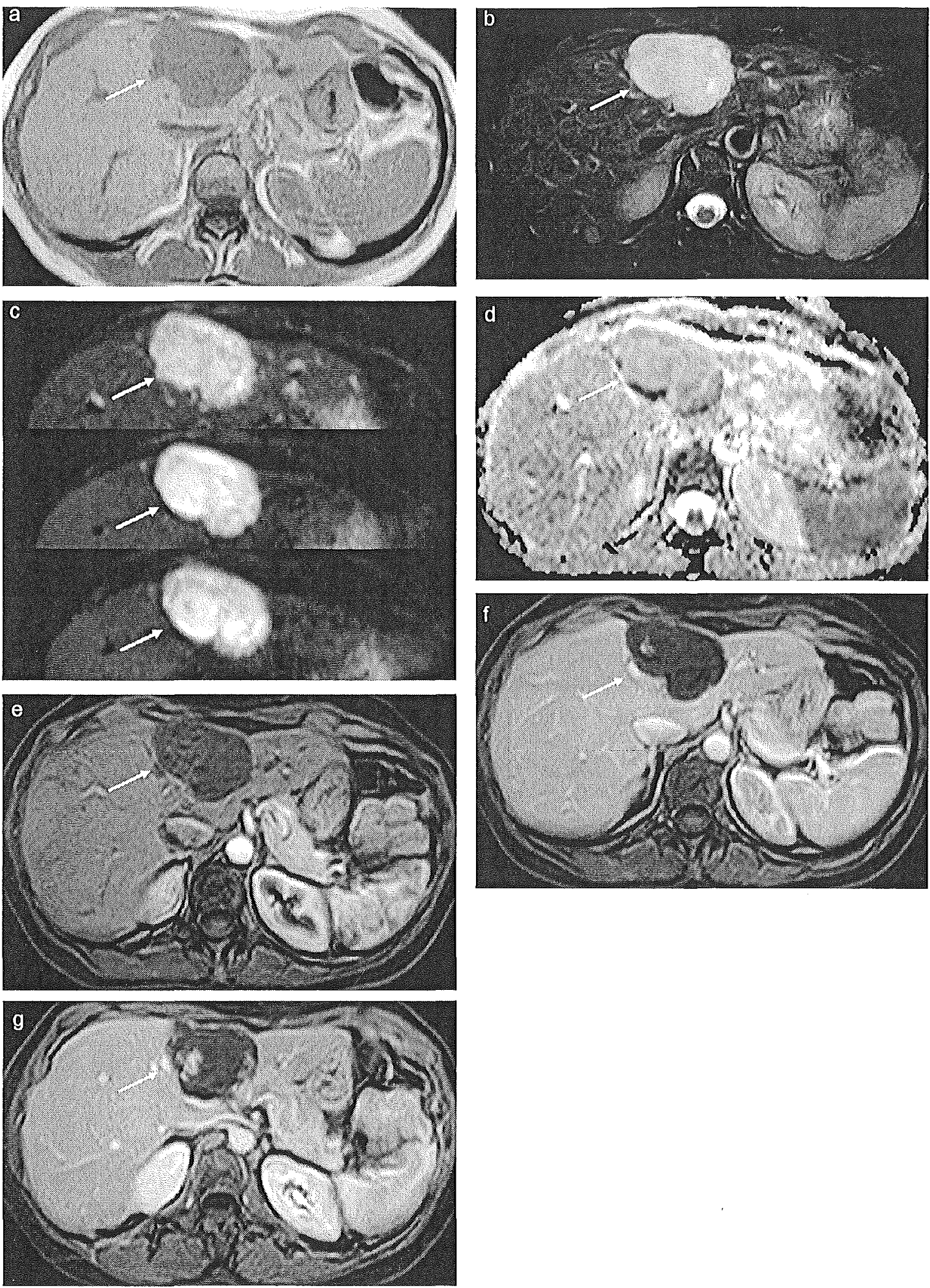

Fig. 5. Hepatic hemangioma with delayed enhancement pattern in a 53-year-old woman with unknown hepatic lesion on ultrasound. (a) On in-phase gradient-recalled echo T1-weighted MR sequence, the hepatic hemangioma appears hypointense (arrow) and (b) on fat-suppressed T2-weighted fast spin-echo MR sequence, the lesion is hyperintense relative to surrounding liver and spleen (arrow). (c) On DW MR sequence, the lesion-to-liver contrast is increased with a $b$ value of $600 \mathrm{~s} / \mathrm{mm}^{2} \mathrm{compared} \mathrm{with} \mathrm{a}$ $b$ value of $0 \mathrm{~s} / \mathrm{mm}^{2}$ (T2 shine-through effect). (d) ADC value of the lesion is $1.73 \pm 0.34 \times 10^{-3} \mathrm{~mm}^{2} / \mathrm{s}$ (arrow). (e-g) On dynamic contrast-enhanced MR sequences, the lesion shows a lack of enhancement in the arterial phase (e) with minimal peripheral enhancement in the portal (f) and equilibrium phases (g) (arrows). 

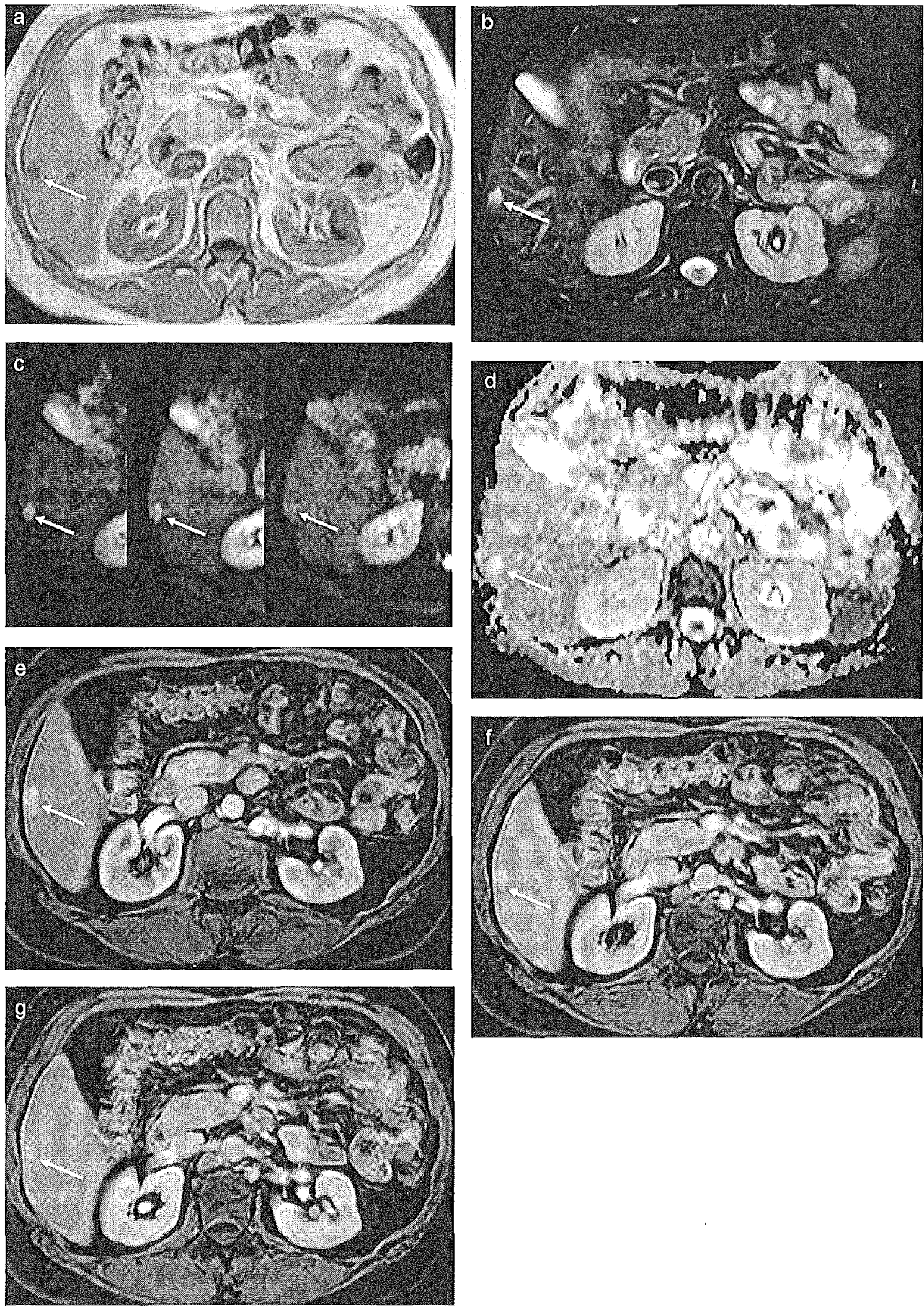

Fig. 6. Hepatic hemangioma with early enhancement pattern in a 43-year-old man with thyroid cancer. (a) On in-phase gradient-recalled echo T1-weighted MR sequence, the hepatic hemangioma appears hypointense (arrow) and (b) on fat-suppressed T2-weighted fast spin-echo MR sequence, the lesion is hyperintense relative to surrounding liver and spleen (spleen partially shown) (arrow). (c) On DW MR sequence, the lesion-to-liver contrast is decreased with a $b$ value of $600 \mathrm{~s} / \mathrm{mm}^{2}$ compared with a $b$ value of $0 \mathrm{~s} / \mathrm{mm}^{2}$. (d) ADC value of the lesion is $2.64 \pm 0.38 \times 10^{-3} \mathrm{~mm}^{2} / \mathrm{s}$ (arrow). (e-g) On dynamic contrast-enhanced MR sequences, the lesion shows immediate homogeneous enhancement at the arterial phase (e) that persists at the portal phase (f) and equilibrium phase (g) (arrows). 

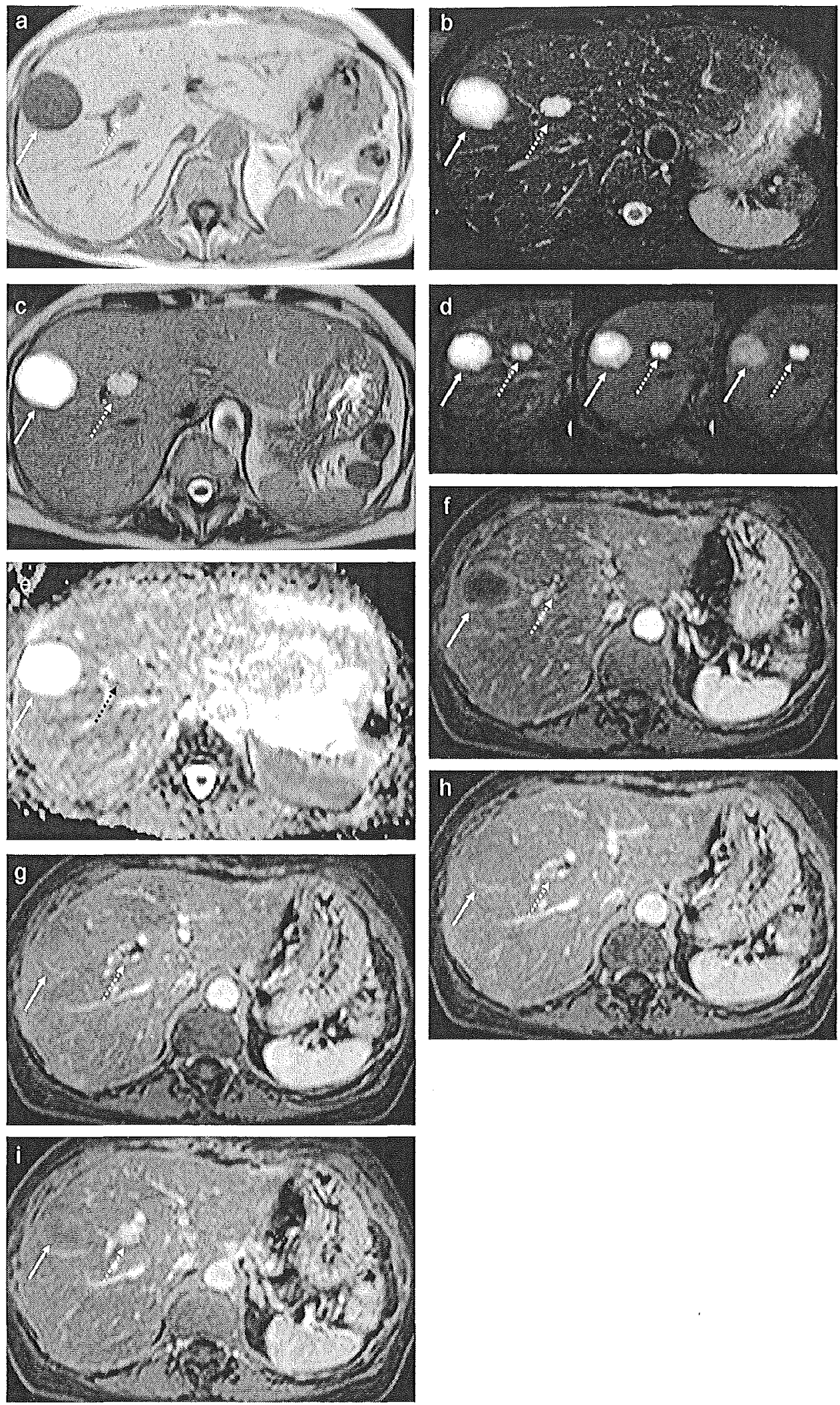

Fig. 7. Typical hepatic hemangioma in a 66-year-old woman with intraductal papillary mucinous neoplasm. (a) On in-phase gradient-recalled echo T1-weighted MR sequence, a hepatic hemangioma (dotted arrow) and a cyst (solid arrow) appear hypointense and (b) on fat-suppressed T2-weighted fast spin-echo MR sequence and (c) single-shot T2-weighted sequences, these lesions are hyperintense relative to surrounding liver and spleen. (d) On DW MR sequence, the lesion-to-liver contrast is decreased with a $b$ value of $600 \mathrm{~s} / \mathrm{mm}^{2}$ compared with a $b$ value of $0 \mathrm{~s} / \mathrm{mm}^{2}$ for the cyst (solid arrow) but not for the hepatic hemangioma (dotted arrow) which has an increased lesion-to-liver contrast with a $b$ value of $600 \mathrm{~s} / \mathrm{mm}^{2}$ (T2 shiné-through effect). (e) ADC value of the hepatic hemangioma is $1.87 \pm 0.37 \times 10^{-3} \mathrm{~mm}^{2} / \mathrm{s}$ (black dotted arrow). (f-i) On dynamic contrast-enhanced MR sequences, the hepatic hemangioma shows early, peripheral, globular, and discontinuous enhancement with progressive centripetal enhancement (dotted arrows) 
$2.44 \pm 0.52 \times 10^{-3} \mathrm{~mm}^{2} / \mathrm{s}, \quad 1.98 \pm 0.5 \times 10^{-3} \mathrm{~mm}^{2} / \mathrm{s}$, respectively, $P=.0002$ )

On multivariate analysis, the only significant factors associated with $\mathrm{T} 2$ shine-through effect were high signal intensity on fat-suppressed T2-weighted fast spin-echo images $(P=.0119)$, the classical or delayed filling hepatic hemangiomas on contrastenhanced sequences $(P<.0001)$ and a low ADC value of the liver $(P=.0114)$.

\section{Discussion}

To our knowledge, this is the first study that has evaluated the frequency and the associated factors of $\mathrm{T} 2$ shine-through effect in patients with hepatic hemangiomas. We have shown that T2 shine-through effect is approximately observed in half of the lesions and in two-thirds of a large patient population with hepatic hemangiomas. As persistent hyperintensity compared with liver parenchyma at high $b$ values is part of the criteria that suggest malignancy, it is important to emphasize this common finding in hepatic hemangiomas [16]. Presence of T2 shine-through effect was even more common in typical hemangiomas (including strong hyperintensity on T2-weighted sequences and classical enhancement) than in other hemangiomas. Indeed, specificity of MR imaging for the diagnosis of hepatic hemangiomas nearly reaches $100 \%$ when typical MR features are seen but we want to stress the fact that DW MR images should be read concurrently with all available MR sequences to avoid misinterpretation [4]. ADC measurement should be helpful for recognizing T2 shine-through effect as lesions that show persistent hyperintensity on high $b$ values related to this phenomenon have high diffusivity on the ADC map and high ADC values. In our series, although mean ADC value was significantly lower in hemangiomas showing T2 shine-through effect, it was still far above the ADC cutoffs $\left(1.4-1.6 \times 10^{-3} \mathrm{~mm}^{2} / \mathrm{s}\right)$ that have been described in the literature for distinguishing benign from malignant liver lesions [10]. Yet, in daily routine ADCs may not be systematically measured and ADC measurement might be problematic in small-sized lesions.

We have found several factors that have been significantly associated with the presence of $\mathrm{T} 2$ shine-through effect. The long T2-relaxation time observed in the vast majority of hemangiomas which is related to the fluid content might explain the high occurrence of $\mathrm{T} 2$ shine-through effect in our series. As a confirmation, T2 shine-through effect was less often observed in hemangiomas which were not as hyperintense as the spleen on fat-suppressed T2-weighted fast spin-echo sequences.

T2 shine-through effect was significantly more often seen in hemangiomas in the right liver lobe than in the left liver lobe and the mean ADC value of hemangiomas in the right liver was significantly lower than that in the left liver. These differences might be attributed to the artifacts caused by cardiac motion predominantly observed in the left lobe of the liver, resulting in higher ADC values over the left liver lobe $[17-20]$.

On multivariate analysis, the pattern of contrast-enhancement was a significant factor and $\mathrm{T} 2$ shine-through effect was more frequent in classical and delayed filling hemangiomas than in rapidly filling hemangiomas. Interestingly, significant differences in ADC values according to the enhancement pattern have been previously shown with the highest mean ADC value in rapidly filling hemangiomas, followed by classical and delayed filling hemangiomas [14]. Hemangiomas are blood-pooled lesions and their different enhancement patterns are related to the collective sizes of their vascular spaces [13]. In rapidly filling hemangiomas, contrast agent fills-in immediately and completely all vascular spaces reflecting high blood flow velocity and therefore high diffusivity. on the contrary, classical and delayed hemangiomas, which are usually larger, contain vascular spaces with decreased perfusion and extensive scar tissue explaining the lower diffusivity than rapidly filling hemangiomas. Hence, we might speculate that persistent signal on high $b$ values in some hemangiomas could correspond in part to true $\mathrm{T} 2$ shine-through effect favored by long T2-relaxation time and in part to differences in diffusivity related to contrast-enhancement, which is confirmed by differences in ADC values.

There were several limitations to this study. First, the retrospective design of our study constituted a classical limitation. However, we included patients with hepatic hemangiomas who had MR imaging in a consecutive manner in order to limit sampling bias. Second, the diagnosis of hepatic hemangiomas was mainly based on imaging features and follow-up period and rarely on tissue sampling obtained by surgery or biopsy (only eleven lesions were histologically confirmed). This is a common limitation with hepatic hemangiomas as $C T$ and/or MR imaging are highly reliable for the diagnosis of hemangiomas. But if only histopathologically confirmed hemangiomas had been taken into account, this would not correspond to clinical practice. Third, we did not separate diffusion and perfusion by means of a wide range of $b$ values and biexponentional fitting and we used in our study a three-point method to calculate ADC values with $b$ values of 0,150 , and $600 \mathrm{~s} / \mathrm{mm}^{2}$, the two-point method being less reliable $[10,11]$. Yet, we wanted to focus on the visual assessment of DW images. Fourth, we could have used higher $b$ values to mitigate the T2 shine-through effect. Yet, the highest $b$ value used in our protocol $\left(600 \mathrm{~s} / \mathrm{mm}^{2}\right)$ reflects the current state of the art recommendations (i.e. $500-750 \mathrm{~s} / \mathrm{mm}^{2}$ ) in liver DW MR imaging $[10,11]$.

In conclusion, T2 shine-through effect is frequently observed in hepatic hemangiomas on high $b$ values DW images, and mostly in classical or delayed filling hemangiomas. Radiologists should be aware of this phenomenon in order to avoid making an erroneous diagnosis of malignancy, particularly when contrast material injection is contraindicated.

\section{Acknowledgement}

We would like to thank Sabrina Doblas, PhD for technical support.

\section{References}

[1] Ishak KG, Rabin L. Benign tumors of the liver. Wed Clin North Am 1975:59:505-1013.

[2] Semelka RC, Solka CM. Hepatic hemangiomas. Magn Reson Imaging Clin $\mathrm{N}$ An $1997: 5: 241-53$.

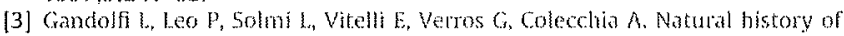
hepatic hematrgiomas: dinical and ultrasound study. Gut 1991;32:677 -80.

[4] Whitney WS Herkens R]. Jefirey RE, at al. Dynamic breath-hold multipla nar spoled gradient-recalled MR imaging with gadohium enhancement for differentiating hepatic hemangionas from malignancies at $1.5 \%$. Radiology $1993: 189: 863 \cdots 70$

[5] Semelka RC. Brown ED, Ascher SM, et al. Hepatic hemangiomas: a multi-institutional study of appearance on $\mathrm{T} 2$ - weighted and serial gadoliniumenlanced gradientedo MR images. Rathology $1994 ; 192: 401 \ldots 6$.

[6] Kim T Fedeste MP Baron Rl Peterson MS Kawanori $Y$ Discrimination of smal hepatic hemangiomas from hypervascular malignant tumors smallet than $3 \mathrm{~cm}$ with three-phase helical CT. Radiology 2001;219:699-706.

17] Vilgrain V. Bonlos L, Vulleme MP, Denys A, Terris B, Menu Y. Imaging of atypical hemangiomes of the liver with pathologic corretation. Radiographics $2000: 20: 379-97$

[8] Hanafusa K, Ohashi I, Himeno Y, Stzuki S, Shibuya H. Hepatic hemancrioma: findings with twomphase CT. Radiology 1995:196:465-9.

[9] Parikn T. Drew S]. Lee VS, et al. Focal liver lesion detection and chatacterization with diflusion-weighted MR imaging: comparison with standard breath-hold 12-weighted imaging, Radiology 2008:246:812-22.

[10] Taonli B, Koh DM. Diffusion-weighed MR imaging of the liver, Radiology $2010,254: 47-66$

[11] Padhani $A R$, Lit $G$, Koh DM, et al. Diffusion-weighted magnetic resonanca maging as a cancer biomarker: consensus and recommendarions. Neoplasia $2009 ; 11: 102-25$ 
[12] Burdette]H, Llster AD, Ricci PE. Acutecerebal infarction: quantification of spindensity and 72 shine-though phenomena on diffusion-weighted MR images. Radiology 1999;212:333-9.

[13] Quina SF, Benjamin GG. Hepatic cavenous hemangiomas: smple diagnostic sign with dynamic bolus CT. Radiology 1992:182:545-8.

14] Coshima $S$ Kanematsu $\mathrm{M}$, Kondo $\mathrm{H}$, et al, Hepate homangioma: correlation of enhancement types with diffusion-weighted MK fndings and apparent diffusion coeficients. Eur ] Radiol $2009 ; 70: 325 \cdots 30$.

[15] Yamashita Y, Ogatal, Urata J, Takahashi M. Cavernous henangona of the liver: 3achologic correlation with dynamic CT findings Ractiology 1997;2033127-5.

[16] Fenerben S, Pauls S, Juchems NS, et al. Piffalls in abdominal diffrsion-weighted inaging: how predictive is restricted water diftusion for malignancy. Am I Reentenol 2009; 193:1070-6.
[17] Mürz P. Facke S, Träber F, van den Brink J5, Gieseke J. Schild HH. Abtomen: diffusion-weighted MR imaging with pulse-triggered single-shot sequences Radiology $2002 ; 224: 258-64$.

[18] Bragel $M$, Holzapta $K$. Ga J, et al Chancterization of focal liver jesions by $A D C$ maxtsemeats using a respiatory triggered diffusion. weighted single-shot echo-planar MR Imaging techoique. Eus Radiol 2008; 18: $477-35$.

[19] Nasu K, Kuroki $Y$, Nawano $S$, ext al. Hepatic metastases: diffusionweighted sensitivity-encoding versus SPlo-enhanced MR imaging. Ratiology $2006 ; 239: 122-30$.

[20] Kwee TC. Takakara $T$, Niwa $T$, at al Inflence of cardiac motion on diffusion.weighted magnetic resonance imaghg of the liver. WAGMA 2009;22: $319 \cdots 25$. 